\title{
СТРУКТУРНО-ФУНКЦИОНАЛЬНЫЕ ПАРАМЕТРЫ СЕРДЕЧНО-СОСУДИСТОЙ СИСТЕМЫ ПРИ ФИБРИЛЛЯЦИИ ПРЕДСЕРДИЙ У БОЛЬНЫХ, ПЕРЕНЕСШИХ ИНСУЛЬТ
}

\section{STRUCTURAL AND FUNCTIONAL PARAMETERS OF THE CARDIOVASCULAR SYSTEM IN PATIENTS WITH ATRIAL FIBRILATION AFTER A STROKE}

\author{
Е.В. Бондаренко, Л.А. Камышникова, О.А. Ефремова, Ю.С. Павлова, А.Н. Паюдис \\ E.V. Bondarenko, L.A. Kamyshnikova, O.A. Efremova, Yu.S. Pavlova, A.N. Payudis
}

\author{
Белгородский государственный национальный исследовательский университет, \\ Россия, 308015, г. Белгород, ул. Победы, 85 \\ Belgorod National Research University, \\ 85 Pobeda St., Belgorod, 308015, Russia \\ E-mail: efremova@bsu.edu.ru
}

\begin{abstract}
Аннотация
Фибрилляция предсердий (ФП) является важной медицинской проблемой в связи с широкой распространенностью и повышенной летальностью. При развитии инсульта у больного возникает пагубная ситуация не только для головного мозга, но и для всего организма, что ведет к ухудшению общего соматического статуса. В данной статье мы проводим анализ данных структурно-функциональных параметров сердечно-сосудистой системы при фибрилляции предсердий у больных, перенесших инсульт, для выявления динамики показателей у пациентов после инсульта. Цель исследования - оценить структурно-функциональные параметры сердечнососудистой системы при фибрилляции предсердий у больных, перенесших инсульт. В основную группу нами были отобраны 28 пациентов с неклапанной фибрилляцией предсердий, ранее перенесших ишемический инсульт. В группу сравнения (30 человек) вошли пациенты с неклапанной ФП без инсульта и транзиторных ишемических атак в анамнезе, сопоставимые по возрасту и полу. Всем пациентам проводили лабораторную диагностику, электрокардиографию, эхокардиографию, ультразвуковое дуплексное сканирование артерий брахиоцефальной зоны, а также заполняли индивидуальную анкету. В результате исследования нами выявлены статистически значимые различия между группами в отношении эхокардиографических параметров (толщина межжелудочковой перегородки, толщина задней стенки левого желудочка (ЛЖ) и индекс массы миокарда) у пациентов, перенесших ишемический инсульт. Показано увеличение риска инсульта при увеличении толщины комплекс интима-медиа $>0,9$ мм. Индекс объема левого предсердия выше нормы свидетельствовал о наличии диастолической дисфункции ЛЖ в обеих группах.
\end{abstract}

\begin{abstract}
Atrial fibrillation (AF) is an important medical problem due to its high prevalence and increased mortality. With the development of a stroke, the patient has a fatal situation not only for the brain, but also for the whole organism, which leads to a deterioration in the general somatic status. In this article, we analyze the structural and functional parameters of the cardiovascular system during AF in patients who have had a stroke, in order to identify the dynamics of indicators in patients after a stroke. The aim of the study was to evaluate the structural and functional parameters of the cardiovascular system in patients with stroke after atrial fibrillation. In the main group, we selected 28 patients with non-valve atrial fibrillation, who had previously suffered an ischemic stroke. The comparison group (30 people) included patients with non-valve AF without stroke and transient ischemic attacks in history, comparable in age and gender. All patients underwent laboratory diagnostics by electrocardiographic and echocardiography, ultrasound duplex scanning of the arteries of the brachiocephalic zone, and also filled out an individual
\end{abstract}


questionnaire. As a result of the study, we revealed statistically significant differences between the groups with respect to echocardiographic parameters (thickness of the interventricular septum, thickness of the posterior wall of the left ventricle (LV) and myocardial mass index) in patients after ischemic stroke. An increase in the risk of stroke with an increase in the thickness of intima media complex $>0.9 \mathrm{~mm}$ is shown. The left atrial volume index is higher than normal in both groups, which indicates the presence of LV diastolic dysfunction.

Ключевые слова: ишемический инсульт, неклапанная фибрилляция предсердий, эхокардиография, ЭхоКГ, структурно-функциональные параметры сердца.

Keywords: ischemic stroke, non-valvular atrial fibrillation, echocardiography, structural and functional parameters of the heart.

\section{Введение}

Фибрилляция предсердий (ФП) является важной медицинской проблемой в связи с широкой распространенностью и повышенной летальностью, увеличивая риск инсульта в 5 раз и на 40-90 \% повышая риск общей смертности [Powers WJ et al., 2018].

По мнению Hannon N. и соавторов, при развитии инсульта у больного возникает пагубная ситуация не только для головного мозга, но и для всего организма, что ведет к ухудшению общего соматического статуса [Hannon et al., 2011].

Одним из факторов риска ишемического инсульта (ИИ) является снижение пиковой скорости кровотока в ушке левого предсердия (УЛП) по данным эхокардиографии (ЭхоКГ) сердца. При этом считается, что показатель ассоциирован с риском внутрипредсердного тромбоза вне зависимости от формы и длительности ФП [Гронтковская и др., 2016].

По данным Голуховой Е.3., при проведении чреспищеводной ЭхоКГ пациентам с ФП выявлено, что параметры гемодинамики сердца, в частности снижение скорости кровотока в УЛП менее $30 \mathrm{~cm} / \mathrm{c}$ и морфологический тип УЛП, являются предикторами внутрисердечного тромбогенеза [Голухова и др., 2017].

Имеются данные, что снижение фракции выброса левого желудочка (ФВ ЛЖ) $<50$ \% и увеличение ЛП более 42 мм увеличивает риск тромбоза УЛП при ФП [Корнелюк и др., 2014].

В основном проводятся ретроспективные исследования для изучения клинических или лабораторных факторов риска тромбообразования у пациентов ФП, перенесших кардиоэмболический инсульт (КЭИ). Описана анатомия и морфология УЛП, доказывающая связь формирования тромба именно в УЛП и осложнений при ФП.

Однако в последние годы многие исследования доказали, что наиболее точным критерием ремоделирования ЛП следует считать индекс объема ЛП (ОЛП) [Gupta et al., 2013]. Было доказано, что именно объем ЛП коррелирует со степенью предсердного фибpoзa [Lupu et al., 2014]. Есть данные по оценке диастолической функции (ДФ) ЛЖ у больных с ФП как о предикторе рецидива аритмии [Caputo, Mondillo, 2012], однако до сих пор не существует точных ЭхоКГ параметров прогнозирования инсульта. Есть исследования по нарушению функции ЛП (станнингу) как предиктору рецидивирования аритмии и возможных КЭИ [Gupta et al., 2013; Caputo, Mondillo, 2012]. «В основе возникновения данного феномена лежат несколько механизмов: избыток кальция в цитоплазме миофибрилл, гибернация миокарда предсердий, обусловленная тахикардией, а также фиброз предсердий» [Громыко, 2018].

При ФП затруднена оценка диастолической дисфункции (ДД) с помощью Эхо-КГ из-за отсутствия предсердного сокращения (пик А) и невозможно выявление типа ДД ЛЖ. Также имеет место постоянное варьирование показателей при каждом сокращении, что делает необходимым повторные измерения с целью получения среднего значения и выявления ДФ ЛЖ [Камышникова, Ефремова, 2009; Nagueh et al., 2009; Powers et al., 2018]. 
Есть возможность оценки ДД с помощью тканевой допплерографии (ТД): скорость движения фиброзного кольца митрального клапана (МК) меряется в фазу раннего наполнения ЛЖ (E') и представляет собой активный компонент диастолы [Maurer et al., 2004]. Отношение E' к пику Е трансмитрального кровотока $\left(\mathrm{E} / \mathrm{E}^{\prime}-\right.$ т. е. отношения скоростей раннедиастолического потока и раннедиастолического движения миокарда) позволяет косвенно оценить давление в полости ЛП. Отношение (E/E' > 15) отражает подъем давления в ЛП и рост давления наполнения ЛЖ и является одним из ключевых параметров оценки ДФ ЛЖ у пациентов с ФП [Leong et al., 2010]. Данный показатель коррелирует с коэффициентом «тау», отражающим уменьшение давления в ЛЖ в период релаксации.

Caputo M. и соавторы считают, что измерение ElE' как критерия оценки ДД ЛЖ должны быть обязательным для ЭхоКГ протокола у лиц с ФП, и тактика лечения больных с ДД ЛЖ и ФП должна включать в себя более длительную антикоагулянтную терапию [Caputo, Mondillo, 2012]. Но все же на сегодняшний день остается много вопросов относительно точности разных методов оценки ДФ ЛЖ у пациентов с ФП.

Анализируя исследования последних лет у пациентов с ФП по ЭхоКГ показателям, выявлено, что единственный фактор риска ишемического инсульта (ИИ) - это наличие систолической дисфункции ЛЖ (СД ЛЖ), соответствующей ФВ ЛЖ < 40 \%. При изучении данных чреспищеводной ЭхоКГ в пользу ишемического инсульта свидетельствуют такие данные, как наличие тромбов в УЛП, уменьшение скорости кровотока в ушке менее $20 \mathrm{~cm} / \mathrm{c}$, наличие спонтанного контрастирования, а также атеросклеротическое поражение аорты [Громыко, 2018].

Выявлено, что у людей с ФП до 60 лет без структурных заболеваний сердца риск ИИ и системных тромбоэмболических осложнений (ТЭО) составляет 1,3 \% на протяжении 15 лет. В отличие от пациентов старше 75 лет, у которых, напротив, риск инсульта оказался очень высоким [Hughes, Lip, 2008].

Таким образом, нам представляется важным оценить структурно-функциональные параметры сердечно-сосудистой системы при фибрилляции предсердий у больных, перенесших инсульт, для выявления ЭхоКГ параметров, увеличивающих риск инсульта и являющихся результатом инсульта.

Цель исследования - оценить структурно-функциональные параметры сердечнососудистой системы при фибрилляции предсердий у больных, перенесших инсульт.

\section{Методы исследования}

В связи с поставленной целью нами были отобраны 28 пациентов с неклапанной фибрилляцией предсердий, ранее перенесших ишемический инсульт из них 18 (64,3\%) мужчин и $10(35,7 \%)$ женщин, средний возраст пациентов составлял 69,6 (62; 74), индекс массы тела (ИМТ) - 29,20 $(24,6 ; 33,2)$ кг/м². Отбор проводился в ОГБУЗ «Городская больница № 2 г. Белгорода» (с января 2018 по декабрь 2019 гг.). В группу сравнения (30 человек) вошли пациенты с неклапанной ФП без инсульта и транзиторных ишемических атак (ТИА) в анамнезе, сопоставимые по возрасту: 19 (63,3 \%) мужчин и 11 (36,7 \%) женщин. Критерии включения: наличие неклапанной ФП; наличие 2 балла при расчете риска ТЭО по шкале CHA2DS2-VASc у мужчин и 3 - у женщин. Из исследования исключались пациенты с клапанной ФП (средне-тяжелым и тяжелым митральным стенозом или механическим протезом клапанов сердца), тиреотоксикозом, онкологическим заболеванием в анамнезе.

Всем пациентам проводили лабораторную диагностику, электрокардиографическое (ЭКГ) и эхокардиографическое исследования (ЭхоКГ), ультразвуковое дуплексное сканирование артерий брахиоцефальной зоны (УЗДС БЦА), а также заполняли индивидуальную анкету, в которой указывались данные анамнеза с указанием даты начала фибрилляции предсердий, перенесенного инсульта, сопутствующей патологии и все лекарственные средства, которые принимает пациент. 
Структурно-функциональные параметры сердца оценивали на ультразвуковом аппарате GE VIVID 7 Vantage (Цифровая ультразвуковая система экспертного класса General Electric Medical Systems) Датчик-мультичастотный 2,5-4 для кардиологических исследований. ЭхоКГ проводили по общепринятой методике в М и В режимах и тканевой допплерографией. Определение фракции выброса ЛЖ (ФВ) по методу Симпсона [Lang et al., 2005].

Оценка «комплекса интима-медиа (КИМ) и процент стеноза сонных артерий (CA) выполнялась на аппарате в соответствии с Российскими национальными рекомендациями по кардиоваскулярной профилактике 2017 года» [Кардиоваскулярная профилактика, 2017]. Определяли толщину КИМ СА (мм) в трех стандартных точках. Повышением считали толщину КИМ $>0,9<1,3$ мм. Локальные утолщения более 1,3 считали свидетельством присутствия атеросклеротической бляшки (АСБ). Оценку степени стеноза сонных артерий проводили с использованием критериев ECST-метод (European Carotid Surgery Method), при котором степень стеноза бифуркации общей сонной артерии вычисляется как отношение разности величины максимального и свободного просвета сосуда к величине максимального диаметра сосуда, выраженное в процентах [European Carotid Surgery Trialists' Collaborative Group, 1991].

Статистическую обработку материала проводили с помощью программы «Statistica 6.0». Применялись методы описательной статистики с определением медианы $(\mathrm{Me})$ и интерквартильный размах с указанием нижнего и верхнего квартилей $(\mathrm{Q} 1-\mathrm{Q} 3)$. Для описания качественных показателей использовали проценты. Все показатели приведены в системе СИ. Для оценки достоверности различий между группами по количественным признакам использовался непараметрический критерий Манна - Уитни. Анализ различия качественных признаков в двух независимых группах осуществлялся при помощи построения таблиц сопряженности с последующим расчетом критерия $\chi^{2}$ Пирсона и ОШ с расчетом $95 \%$ ДИ. Нормальность распределения проверялась при помощи теста Шапиро Уилка, было выявлено ненормальное распределение. Достоверными считали изменения показателей при $\mathrm{p}<0,05$.

\section{Результаты и их обсуждение}

Среди обследованных пациентов были выявлены сопутствующие заболевания в анамнезе: артериальная гипертензия (АГ), ишемическая болезнь сердца (ИБС) и хроническая сердечная недостаточность $(\mathrm{XCH})$. Средняя длительность заболевания ФП составляла $4,7(2,6 ; 6,4)$ года в основной группе и $3,8(2,0 ; 5,1)$ года в группе сравнения $(\mathrm{p}=0,07)$. Инсульт был перенесен в диапазоне от 4 до 19 месяцев назад.

В основной группе было $9(32,13 \%)$ пациентов с пароксизмальной формой ФП, $10(35,7 \%)$ - с персистирующей и $9(32,13 \%)$ - с постоянной формой фибрилляции предсердий.

Нами выявлена недостаточная приверженность к приему антикоагулянтов. Только $12(42,87 \%)$ пациентов принимали прямые оральные антикоагулянты (ПОАК), $5(17,86 \%)$ - принимали варфарин и $10(35,7 \%)$ - указывали только на прием ацетилсалициловой кислоты (АСК) и/или клопидогреля. Совсем не принимал антитромботических препаратов 1 (3,57 \%) пациент. При этом на вопрос о том, были ли назначены антикоагулянты, почти все пациенты (92,8 \%) ответили положительно, оставшиеся 2 не могли вспомнить о назначении этих препаратов.

При исследовании предшествующей антитромботической терапии в группе сравнения были следующие показатели: АСК и/или клопидогрель получали 15 (50\%) человек, варфарин - $8(26,7) \%$, НОАК - $7(23,3 \%)$.

Наши данные сопоставимы с результатами исследования Карпова А.Ю., где АСК получали 50,6 \%, варфарин - 23,1 \%, дабигатран - 8,3 \%, ривароксабан - 19,4 \% и апиксабан - 4,3\% больных [Карпов, 2018]. 
У пациентов в основной группе средний балл по шкале CHA2DS2-VASc был $4,5(3 ; 5)$ а в группе сравнения - 2,75 $(2 ; 4), \mathrm{p}=0,068$.

Есть исследование у 281 больного с ФП из Красноярска [Тучков и др., 2010]. По данным их регистра, у пациентов средний балл по шкале CHA2DS2-VASc составил $3,6(2,0-5,0)$, что также сопоставимо с полученными данными из международного регистра GARFIELD, в котором средний балл 3,2, и Европейского (PREFER in AF) - 3,4 балла [Kakkar et al., 2012; Kirchhof et al., 2014].

В настоящее время нет единого точного эхокардиографического параметра, который используется для постановки диагноза диастолической дисфункции ЛЖ у пациентов с ФП. Рекомендовано использовать эхокардиографические обследования, включающие комплексный анализ двухмерной-эхокардиографии, допплер-эхокардиографии, а также цветной тканевой допплерографии [Nagueh et al., 2009; Громыко, Сайганов, 2017].

Таблица 1

Table 1

Динамика структурно-функциональных параметров сердечно-сосудистой системы при фибрилляции предсердий у больных, перенесших инсульт

Dynamics of structural and functional parameters of the cardiovascular system in patients with atrial fibrilation after a stroke

\begin{tabular}{|c|c|c|c|}
\hline Показатель & $\begin{array}{c}\text { Основная группа } \\
(\mathrm{n}=28)\end{array}$ & $\begin{array}{c}\text { Группа сравнения } \\
(\mathrm{n}=30)\end{array}$ & $\mathrm{p}$ \\
\hline Толщина МЖП, см & $1,19(1,1 ; 1,25)$ & $1,09(1,0 ; 1,19)$ & 0,019 \\
\hline Толщина ЗСЛЖ, см & $1,14(1,05 ; 1,24)$ & $1,09(1,01 ; 1,18)$ & 0,038 \\
\hline ИММЛЖ, г/м² & $123,3(117,1 ; 129,4)$ & $107,4(101,6 ; 115,4)$ & 0,041 \\
\hline КДРЛЖ, см & $4,81(4,21 ; 5,48)$ & $4,76(4,1 ; 5,38)$ & 0,28 \\
\hline КСРЛЖ, см & $3,42(2,67 ; 4,12)$ & $3,39(2,62 ; 4,1)$ & 0,19 \\
\hline ФВ ЛЖ по Симпсон, \% & $53,4(44,8 ; 61,5)$ & $55,12(45,9 ; 63,4)$ & 0,24 \\
\hline Ширина ЛП, см & $4,26(3,65 ; 4,81)$ & $4,23(3,69 ; 4,76)$ & 0,31 \\
\hline Длина ЛП, см & $6,02(5,08 ; 6,9)$ & $5,86(5,03 ; 6,61)$ & 0,12 \\
\hline Объем ЛП, мл & $73,71(49,6 ; 87,2)$ & $71,2(48,1 ; 86,3)$ & 0,26 \\
\hline Индекса объема ЛП, мл/м² & $39,9(34,8 ; 44,7)$ & $37,6(31,4 ; 43,5)$ & 0,11 \\
\hline Е/Е', ед & $14,6(11,2 ; 17,4)$ & $13,8(10,1 ; 16,2)$ & 0,14 \\
\hline Толщина КИМ СА, мм & $1,03(0,97 ; 1,1)$ & $0,91(0,84 ; 1,05) \pm 0,15$ & 0,021 \\
\hline
\end{tabular}

Из таблицы 1 следует, что при сравнении основной группы и группы сравнения выявлены достоверные статистически различия в следующих показателях: толщина межжелудочковой перегородки (МЖП) - 1,19 $(1,1 ; 1,25)$ в группе пациентов с инсультом, а не перенесших ИИ - $1,09(1,0 ; 1,19)$ см $(\mathrm{p}=0,019)$, толщина задней стенки ЛЖ (ЗСЛЖ) больше в основной группе $1,14(1,05 ; 1,24)$ против $1,09(1,01 ; 1,18)$ см в группе сравнения

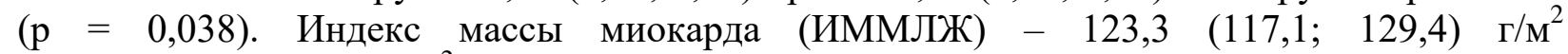
и $107,4(101,6 ; 115,4)$ г $\mathrm{M}^{2}$ в группе сравнения $(\mathrm{p}=0,041)$. Это свидетельствует о гипертрофии ЛЖ в основной группе.

Размеры ЛЖ достоверно не различались: конечный диастолический размер ЛЖ (КДРЛЖ), конечный систолический размер ЛЖ (КСР ЛЖ). Фракция выброса левого желудочка также не имела достоверных различий, в группе больных с инсультом составила $53,4(44,8 ; 61,5) \%$ и 55,12 $(45,9 ; 63,4) \%$ в группе пациентов без инсульта $(p=0,24)$.

Ширина ЛП в первой группе составила $4,26(3,65 ; 4,81)$ см, а во второй группе $4,23(3,69 ; 4,76)$ см; без статистически значимых отличий. Аналогичные результаты получены по объему ЛП - 73,71 $(49,6 ; 87,2)$ мл в основной группе и $71,2(48,1 ; 86,3)$ мл в группе контроля $(\mathrm{p}=0,26)$.

Индекс объема ЛП выше нормы был в обеих группах, что говорит о наличии ДД ЛЖ в обеих группах - 39,9 $(34,8 ; 44,7)$ мл/м² в основной группе и $37,6(31,4 ; 43,5)$ мл/м² 
в группе контроля $(\mathrm{p}=0,11)$. Исследование Gupta S. и соавторов доказали, что наиболее точным критерием ремоделирования ЛП следует считать индекс ОЛП [Gupta et al., 2013]. Также доказано, что именно объем ЛП коррелирует со степенью предсердного фиброза [Lupu et al., 2014], однако до сих пор не существует точных ЭхоКГ параметров прогнозирования инсульта.

Отношения скоростей раннедиастолического потока и раннедиастолического движения миокарда Е/Е' в основной группе - $14,6(11,2 ; 17,4)$ ед., в группе контроля $13,8(10,1 ; 16,2)$ ед. не отличались достоверно. При наличии ФП, безусловно, страдает ДФ ЛЖ, но ее оценка на фоне аритмии затруднена [Баймуканов и др., 2017; Громыко, Сайганов, 2017].

Статистически значимо отличалась толщина КИМ в основной группе $1,03(0,97 ; 1,1)$ мм и $0,91(0,84 ; 1,05)$ мм в группе сравнения $(\mathrm{p}=0,021)$, что свидетельствует о повышенной толщине стенки артерии у пациентов и, по нашему мнению, является не следствием инсульта, а наоборот, инсульт мог быть в результате утолщения КИМ артерий брахиоцефальной зоны. В группе пациентов с ИИ толщина КИМ превышала принятое за норму в сонных артериях 0,9 мм. В основной группе таких пациентов было 19 человек $(67,8 \%)$, а в группе сравнения - только 7 пациентов $(23,3 \%) ; p=0,01$.

Анализируя ЭКГ данные, было выявлено, что признаки гипертрофии ЛЖ наблюдались у 16 пациентов (57,1\%) в основной группе и у 12 пациентов (40\%) в группе сравнения, $\mathrm{p}=0,043$.

Мы провели оценку ассоциации показателей с риском развития инсульта. Результаты расчета отношения шансов при проведении однофакторного анализа представлены в таблице 2.

Таблица 2

Table 2

Отношение шансов показателей с риском развития инсульта

The odds ratio of indicators of the stroke risk

\begin{tabular}{|l|c|c|}
\hline \multicolumn{1}{|c|}{ Фактор } & ОШ & 95 \%ДИ \\
\hline ИММЛЖ по данным ЭхоКГ & 2,596 & $1,253-5,712$ \\
\hline Утолщение КИМ СА & 5,534 & $2,629-11,612$ \\
\hline
\end{tabular}

Из таблицы 2 видно, что значимая ассоциация с риском инсульта выявлена по ИММЛЖ при ЭхоКГ (свидетельство гипертрофии левого желудочка) и по толщине КИМ СА.

Наши данные соотносятся с результатами исследования Николина Д.Ю. с соавторами. По их мнению, ГЛЖ по данным ЭхоКГ ассоциируется с повышением риска ишемического инсульта [Николин, 2018].

\section{Заключение}

Таким образом, в результате исследования нами выявлены статистически значимые различия между группами в отношении эхокардиографических параметров. Показатели, характеризующие толщину межжелудочковой перегородки, толщину задней стенки левого желудочка и индекс массы миокарда левого желудочка, были значимо выше у пациентов, перенесших ишемический инсульт.

Показано увеличение риска инсульта при увеличении толщины комплекс интимамедиа сонных артерий более 0,9 мм и увеличении индекса массы миокарда левого желудочка. В нашем исследовании выявлена достоверно большая частота встречаемости пациентов, у которых КИМ превышала норму в группе с ишемическим инсультом 67,8 \%, а в группе сравнения - только $23,3 \%$.

Индекс объема левого предсердия был выше нормы в обеих группах, $(39,9(34,8 ; 44,7)$ мл/м² в основной группе и $37,6(31,4 ; 43,5)$ в группе контроля, $\mathrm{p}=0,11)$, 
что говорит о наличии диастолической дисфункции левого желудочка при фибрилляции предсердий. Ведь именно объем левого предсердия коррелирует со степенью предсердного фиброза и фибрилляцией предсердий. Однако нами не выявлено статистически значимых различий по индексу объема левого предсердия у пациентов с фибрилляцией предсердий перенесших инсульт и без него.

Конфликт интересов: авторы заявляют об отсутствии конфликта интересов.

\section{Список литературы}

1. Баймуканов А.М., Хамнагадаев И.А., Гендлин Г.Е., Никитин И.Г. 2017. Нарушение диастолической функции сердца при фибрилляции предсердий. Российский медицинский журнал. 23 (2): 101-106. DOI: 10.18821/0869-2106-2017-23-2-101-106.

2. Голухова Е.3., Громова О.И., Аракелян М.Г., Булаева Н.И., Жолбаева А.З., Машина Т.В., Джанкетова В.С., Шляппо М.А. 2017. Предикторы тромбоза ушка левого предсердия и тромбоэмболических осложнений у больных с фибрилляцией предсердий без сопутствующей клапанной патологии и ишемической болезни сердца. Креативная кардиология. 11 (3): 262-72. DOI: 10.24022/1997-3187-2017-11-3-262-272.

3. Громыко Т.Ю. 2018. Динамика структурно-функциональных характеристик левого предсердия в прогнозировании рецидивов фибрилляции предсердий. Автореф. дис. ... канд. медицинских наук. Санкт-Петербург, $28 \mathrm{c.}$

4. Громыко Т.Ю., Сайганов С.А. 2017. Динамика диастолической функции левого желудочка у пациентов с фибрилляцией предсердий при различных способах восстановления синусового ритма. Медицинский Совет. 12: 202-208. DOI:10.21518/2079-701X-2017-12-202-208.

5. Гронтковская А.В., Боровков Н.Н., Аминева Н.В. 2016. Артериальная гипертензия как фактор риска внутрипредсердного тромбоза у больных с фибрилляцией предсердий неклапанной этиологии. Клиническая медицина. 94 (6): 433-438. DOI: 10.18821/0023-2149-2016-94-6-433-438.

6. Ефремова О.А., Клеткина А.С., Камышникова Л.А., Беляева С.С. 2013. Применение антикоагулянтов у больных с фибрилляцией предсердий и пороками сердца. Научные ведомости Белгородского государственного университета. Серия: Медицина. Фармация.25 (168): 118-121.

7. Камышникова Л.А., Ефремова О.А. 2009. Диастолическая дисфункция при хронической сердечной недостаточности - основные диагностические параметры и критерии тяжести. Научные ведомости Белгородского государственного университета. Серия: Медицина. Фармация. 4 (59): 9-13.

8. Кардиоваскулярная профилактика 2017. Российские национальные рекомендации. 2018. Российский кардиологический журнал. (6): 7-122. DOI: 10.15829/1560-4071-2018-6-7-122.

9. Карпов Ю.А. 2018. Исследование безопасности и эффективности апиксабана у пациентов с неклапанной фибрилляцией предсердий в реальной клинической практике в России. Атмосфера. Новости кардиологии. 4. 3-12.

10. Корнелюк И.В., Рабцевич В.А., Корнелюк О.М. 2014. Эхокардиографические предикторы тромбоза ушка левого предсердия у пациентов с персистирующей фибрилляцией предсердий. Анналы аритмологии. 11(3): 170-176. DOI: 10.15275/annaritmol.2014.3.5.

11. Николин Д.Ю., Фокина Е.Г., Грачев В.Г., Липченко А.А., Архипов М.В. 2018. Дополнительные критерии стратификации риска ишемического инсульта у пациентов с фибрилляцией предсердий и одним не связанным с полом баллом по шкале CHA2DS2-VASc обусловленным артериальной гипертонией. Уральский медицинский журнал. 3 (158): 5-11. DOI: 10.25694/URMJ.2018.03.030.

12. Тучков А.А., Гоголашвили Н.Г., Яскевич Р.А. 2018. Состояние и адекватность антикоагулянтной терапии при фибрилляции предсердий в клинической практике. Лечащий врач 7: 7-10.

13. Caputo M., Mondillo S. 2012. Echocardiography in the prediction of atrial fibrillation recurrence: a review. Journal of Atrial Fibrillation. 5 (2): 23-29. DOI:10.4022/jafib.675.

14. European Carotid Surgery Trialists' Collaborative Group. 1991. MRC European Carotid Surgery Trial: interim results for symptomatic patients with severe (70-99\%) or with mild (0-29\%) carotid stenosis. Lancet. 337: 1235-1243.

15. Gupta S., Matulevicius S.A., Ayers C.R., Berry J.D., Patel P.C., Markham D.W., Levine B.D., Chin K.M., de Lemos J.A., Peshock R.M., Drazner M.H. 2013. Left atrial structure and function 
and clinical outcomes in the general population. Eur. Heart. J. 34: 278-285. DOI:10.1093/eurheartj/ehs188.

16. Hannon N., Callaly E.L., Moore A.F., Moore A.F., Chróinín D.N., Sheehan O.C., Marnane M.J., Merwick Á., Kyne L., Duggan J., McCormack P.M.E., Dolan E., Crispino-O'Connell G., Harris D., Horgan G., Williams D., Kellyet P.J. 2011. Improved late survival and disability after stroke with therapeutic anticoagulation for atrial fibrillation: a population study. Stroke. 42: 2503-2508. DOI:10.1161/STROKEAHA.110.602235.

17. Hughes M., Lip G.Y. 2008. Stroke and thromboembolism in atrial fibrillation: a systematic review of stroke risk factors, risk stratification schema and cost effectiveness data. Thromb Haemost. 99: 295-304. DOI: 10.1160/TH07-08-0508.

18. Kakkar A.K., Mueller I., Bassand J.P., Fitzmaurice D.A., Goldhaber S.Z., Goto S., Haas S., Hacke W., Lip G.Y., Mantovani L.G., Verheugt F.W., Jamal W., Misselwitz F., Rushton-Smith S., Turpie A.G. 2012. International longitudinal registry of patients with atrial fibrillation at risk of stroke: Global Anticoagulant Registry in the FIELD (GARFIELD). American Heart Journal. 163 (1): 13-9. e1. DOI: 10.1016/j.ahj.2011.09.011.

19. Kirchhof P., Ammentorp B., Darius H., De Caterina R., Le Heuzey J.Y., Schilling R.J., Schmitt J., Zamorano J.L. 2014. Management of atrial fibrillation in seven European countries after the publication of the 2010 Guidelines on atrial fibrillation: primary results of the prevention of the thromboembolic events - European Registry in Atrial Fibrillation (PREFER in AF). Europace. 16 (1): 6-14. DOI:10.1093/europace/eut263.

20. Leong D.P., De Pasquale C.G., Selvanayagam J.B. 2010. Heart failure with normal ejection fraction: the complementary roles of echocardiography and CMR imaging. J. Am. Coll. Cardiol. Img. 3: 409-420. DOI:10.1016/j.jcmg.2009.12.011.

21. Lupu S., Mitre A., Dobreanu D. 2014. Left atrium function assessment by echocardiography - physiological and clinical implications. Med. Ultrason. 16 (2): 152-161. DOI: 10.11152/mu.201.3.2066.162.sl1am2.

22. Maurer M.S., Spevack D., Burkhoff D., Kronzon I. 2004. Diastolic dysfunction can it be diagnosed by doppler echocardiography? J. Am. Coll. Cardiol. 44: 1543-1549. DOI: 10.1016/j.jacc.2004.07.034.

23. Nagueh S.F., Appleton C.P., Gillebert T.C. 2009. Recommendations for the evaluation of left ventricular diastolic function by echocardiography. Eur. J. Echocardiogr. 10: 165-193.

24. Powers W.J., Rabinstein A.A., Ackerson T. Adeoye O.M., Bambakidis N.C., Becker K., Biller J., Brown M., Demaerschalk B.M., Hoh B., Jauch E.C., Kidwell C.S., Leslie-Mazwi T.M., Ovbiagele B., Scott P.A., Sheth K.N., Southerland A.M., Summers D.V., Tirschwell D.L. 2018. Guidelines for the early management of patients with acute ischemic stroke. A guideline for healthcare professionals from the American Heart Association / American Stroke Association. Stroke. 49: e46-e110. DOI: 10.1161/STR.0000000000000158.

\section{References}

1. Baymukhanov A.M., Khamnagadaev I.A., Gendlin G.E., Nikitin I.G. 2017. Narushenie diastolicheskoj funkcii serdca pri fibrillyacii predserdij [The disorder of diastolic function of heart under fibrillation of atria]. Rossiiskii meditsinskii zhurnal [Medical Journal of the Russian Federation, Russian journal]. 23(2): 101-106. DOI: 10.18821/0869-2106-2017-23-2-101-106.

2. Golukhova E.Z., Gromova O.I., Arakelyan M.G., Bulaeva N.I., Zholbaeva A.Z., Mashina T.V., Dzhanketova V.S., Shlyappo M.A. 2017. Prediktory tromboza ushka levogo predserdiya i tromboembolicheskih oslozhnenij u bol'nyh s fibrillyaciej predserdij bez soputstvuyushchej klapannoj patologii i ishemicheskoj bolezni serdca [Risk factors of left atrial thrombus and/or thromboembolism in patients with nonvalvular, nonishemic atrial fibrillation]. Kreativnaya Kardiologiya [Creative Cardiology], 11 (3): 262-72. DOI: 10.24022/1997-3187-2017-11-3-262-272.

3. Gromyko T.Y. 2018. Dinamika strukturno-funkcional'nyh harakteristik levogo predserdiya v prognozirovanii recidivov fibrillyacii predserdij [The dynamics of the structural and functional characteristics of the left atrium in predicting the recurrence of atrial fibrillation]. Abstract. dis. ... cand. med. sciences. Sankt-Peterburg, 28 p.

4. Gromyko T.Y., Sayganov S.A. 2017. Dinamika diastolicheskoj funkcii levogo zheludochka u pacientov s fibrillyaciej predserdij pri razlichnyh sposobah vosstanovleniya sinusovogo ritma [The dynamics 
of diastolic function of the left ventricle at patients with atrial fibrillation at various]. Meditsinskiy sovet [Medical Council], 12: 202-208 (in Russian). https://doi.org/10.21518/2079-701X-2017-12-202-208.

5. Grontkovskaya A.V., Borovkov N.N., Amineva N.V. 2016. Arterial'naya gipertenziya kak faktor riska vnutripredserdnogo tromboza u bol'nyh s fibrillyaciej predserdij neklapannoj etiologii [Arterial hypertension as a risk factor of intra-atrial thrombosis in patients with atrial fibrillation of nonvalvular etiology. Klin. Med.], 94 (6): 433-438. DOI 10.18821/0023-2149-2016-94-6-433-438.

6. Efremova O.A., Kletkina A.S., Kamyshnikova L.A., Belyaeva S.S. 2013. Primenenie antikoagulyantov u bol'nyh s fibrillyaciej predserdij i porokami serdca [The use of anticoagulants in patients with atrial fibrillation and heart defects]. Nauchnye vedomosti Belgorodskogo gosudarstvennogo universiteta. Seriya: Medicina. Farmaciya. 25 (168): 118-121.

7. Kamyshnikova L.A., Efremova O.A. 2009. Diastolicheskaya disfunkciya pri hronicheskoj serdechnoj nedostatochnosti - osnovnye diagnosticheskie parametry i kriterii tyazhesti [Diastolic dysfunction in chronic heart failure - basic diagnostic parameters and criteria of severity]. Nauchnye vedomosti Belgorodskogo gosudarstvennogo universiteta. Seriya: Medicina. Farmaciya. 4 (59): 9-13.

8. Kardiovaskulyarnaya profilaktika 2017. Rossijskie nacional'nye rekomendacii. 2018 [Cardiovascular prevention 2017. National guidelines]. 2018. Rossijskij kardiologicheskij zhurnal [Russian Journal of Cardiology], (6): 7-122 (in Russian). https://doi.org/10.15829/1560-4071-2018-6-7-122.

9. Karpov Yu.A. 2018. Issledovanie bezopasnosti i effektivnosti apiksabana u pacientov s neklapannoj fibrillyaciej predserdij v real'noj klinicheskoj praktike v Rossii [The Study of Safety and Efficacy of Apixaban in Patients with Nonvalvular Atrial Fibrillation in Real Clinical Practice in Russia]. Atmosfera. Novosti kardiologii [Atmosphere. Cardiology News], 4: 3-12.

10. Karnialiuk I.U., Rabtsevich V.A., Karnialiuk A.M. 2014. Ekhokardiograficheskie prediktory tromboza ushka levogo predserdiya u pacientov s persistiruyushcheĭ fibrillyacieĭ predserdij [Echocardiography predictors of the left atrial appendage thrombus in patients with persistent atrial fibrillation]. Annaly aritmologii, 11 (3). 170-176. DOI: 10.15275/annaritmol.2014.3.5.

11. Nicolin D.Y., Fokina E.G., Grachev V.G., Lipchenko A.A., Arkhipov M.V. Dopolnitel'nye kriterii stratifikacii riska ishemicheskogo insul'ta u pacientov $\mathrm{s}$ fibrillyaciej predserdij $\mathrm{i}$ odnim ne svyazannym s polom ballom po shkale CHA2DS2-VASs obuslovlennym arterial'noj gipertoniej. 2018 [Additional criteria for stratifying the ischemic stroke risk in patients with atrial fibrillation and one nonsex-related score on the CHA2DS2-VASC scale due to arterial hypertension]. Ural'skij medicinskij zhurnal [Ural Medical Journal], 3 (159): 5-11. DOI: 10.25694/URMJ.2018.03.030.

12. Tuchkov A.A., Gogolashvili N.G., Yaskevich R.A. 2018 [State and adequacy of anticoagulant therapy in fibrillation of precurities in clinical practice]. Lechashchiy vrach [Attending doctor], 7: 7-10.

13. Caputo M., Mondillo S. 2012. Echocardiography in the prediction of atrial fibrillation recurrence: a review. Journal of Atrial Fibrillation. 5 (2): 23-29. DOI:10.4022/jafib.675.

14. European Carotid Surgery Trialists' Collaborative Group. 1991. MRC European Carotid Surgery Trial: interim results for symptomatic patients with severe (70-99\%) or with mild (0-29\%) carotid stenosis. Lancet. 337: 1235-1243.

15. Gupta S., Matulevicius S.A., Ayers C.R., Berry J.D., Patel P.C., Markham D.W., Levine B.D., Chin K.M., de Lemos J.A., Peshock R.M., Drazner M.H. 2013. Left atrial structure and function and clinical outcomes in the general population. Eur. Heart. J. 34: 278-285. DOI:10.1093/eurheartj/ehs188.

16. Hannon N., Callaly E.L., Moore A.F., Moore A.F., Chróinín D.N., Sheehan O.C., Marnane M.J., Merwick Á., Kyne L., Duggan J., McCormack P.M.E., Dolan E., Crispino-O'Connell G., Harris D., Horgan G., Williams D., Kellyet P.J. 2011. Improved late survival and disability after stroke with therapeutic anticoagulation for atrial fibrillation: a population study. Stroke. 42: 2503-2508. DOI:10.1161/STROKEAHA.110.602235.

17. Hughes M., Lip G.Y. 2008. Stroke and thromboembolism in atrial fibrillation: a systematic review of stroke risk factors, risk stratification schema and cost effectiveness data. Thromb Haemost. 99: 295-304. DOI: 10.1160/TH07-08-0508.

18. Kakkar A.K., Mueller I., Bassand J.P., Fitzmaurice D.A., Goldhaber S.Z., Goto S., Haas S., Hacke W., Lip G.Y., Mantovani L.G., Verheugt F.W., Jamal W., Misselwitz F., Rushton-Smith S., Turpie A.G. 2012. International longitudinal registry of patients with atrial fibrillation at risk of stroke: Global Anticoagulant Registry in the FIELD (GARFIELD). American Heart Journal. 163 (1): 13-9. e1. DOI: 10.1016/j.ahj.2011.09.011. 
19. Kirchhof P., Ammentorp B., Darius H., De Caterina R., Le Heuzey J.Y., Schilling R.J., Schmitt J., Zamorano J.L. 2014. Management of atrial fibrillation in seven European countries after the publication of the 2010 Guidelines on atrial fibrillation: primary results of the prevention of the thromboembolic events - European Registry in Atrial Fibrillation (PREFER in AF). Europace. 16 (1): 6-14. DOI:10.1093/europace/eut263.

20. Leong D.P., De Pasquale C.G., Selvanayagam J.B. 2010. Heart failure with normal ejection fraction: the complementary roles of echocardiography and CMR imaging. J. Am. Coll. Cardiol. Img. 3: 409-420. DOI:10.1016/j.jcmg.2009.12.011.

21. Lupu S., Mitre A., Dobreanu D. 2014. Left atrium function assessment by echocardiography - physiological and clinical implications. Med. Ultrason. 16 (2): 152-161. DOI: 10.11152/mu.201.3.2066.162.s11am2.

22. Maurer M.S., Spevack D., Burkhoff D., Kronzon I. 2004. Diastolic dysfunction can it be diagnosed by doppler echocardiography? J. Am. Coll. Cardiol. 44: 1543-1549. DOI: 10.1016/j.jacc.2004.07.034.

23. Nagueh S.F., Appleton C.P., Gillebert T.C. 2009. Recommendations for the evaluation of left ventricular diastolic function by echocardiography. Eur. J. Echocardiogr. 10: 165-193.

24. Powers W.J., Rabinstein A.A., Ackerson T. Adeoye O.M., Bambakidis N.C., Becker K., Biller J., Brown M., Demaerschalk B.M., Hoh B., Jauch E.C., Kidwell C.S., Leslie-Mazwi T.M., Ovbiagele B., Scott P.A., Sheth K.N., Southerland A.M., Summers D.V., Tirschwell D.L. 2018. Guidelines for the early management of patients with acute ischemic stroke. A guideline for healthcare professionals from the American Heart Association / American Stroke Association. Stroke. 49: e46-e110. DOI: 10.1161/STR.0000000000000158.

\section{Ссылка для цитирования статьи Link for article citation}

Бондаренко Е.В., Камышникова Л.А., Ефремова О.А., Павлова Ю.С., Паюдис А.Н. 2020. Структурно-функциональные параметры сердечно-сосудистой системы при фибрилляции предсердий у больных, перенесших инсульт. Актуальные проблемы медицины, 43 (2): 196-205. DOI 10.18413/2687-0940-2020-43-2-196-205.

Bondarenko E.V., Kamyshnikova L.A., Efremova O.A., Pavlova Yu.S., Payudis A.N. 2020. Structural and functional parameters of the cardiovascular system in patients with atrial fibrilation after a stroke. Challenges in Modern Medicine, 43 (2): 196-205 (in Russian). DOI 10.18413/2687-0940-2020-43-2-196-205. 